\title{
Aufgaben, Möglichkeiten und Grenzen der Betreuung von Patienten am Lebensende
}

\author{
M. Zimmermann-Acklin
}

1 SAMW. Behandlung und Betreuung von zerebral schwerstgeschädigten Langzeitpatienten. Schweiz Ärztezeitung 2003; 84(5):205-9.
Korrespondenz:

Markus Zimmermann-Acklin Institut für Sozialethik (ISE)

Universität Luzern

Gibraltarstrasse 3

Postfach 7763

CH-6000 Luzern 7
Die Betreuung sterbender Patienten stellt Ärzte und andere Mitglieder des Betreuungsteams nicht selten vor grosse Herausforderungen. Hoffnungen, Erwartungen und unterschiedliche Vorstellungen von einem guten Sterben bei Patienten und deren Angehörigen, politische Debatten mit gegensätzlichen rechtlichen und gesellschaftlichen Forderungen, nicht zuletzt die stete Ausweitung medizinisch-technischer Behandlungsmöglichkeiten machen es zur Notwendigkeit, sich mit diesen Herausforderungen insbesondere aus standesethischer Perspektive kontinuierlich auseinanderzusetzen. Die Diskussion und Veröffentlichung der letzten medizinisch-ethischen Richtlinien zur Betreuung Sterbender liegen bereits einige Jahre zurück. Angesichts dieser Situation hat sich die SAMW dazu entschlossen, die «Medizinisch-ethischen Richtlinien für die ärztliche Betreuung sterbender und zerebral schwerst geschädigter Patienten» aus dem Jahre 1995 in zwei getrennten Richtlinien grundlegend $\mathrm{zu}$ überarbeiten. Während die Richtlinien zur «Behandlung und Betreuung von zerebral schwerstgeschädigten Langzeitpatienten» demnächst in ihrer endgültigen Fassung publiziert werden [1], legt die SAMW die neuen Richtlinien zur Betreuung von Patienten am Lebensende im folgenden zur Vernehmlassung vor.

Die Richtlinien wurden von einer interdisziplinär zusammengesetzten Subkommission in einem eineinhalbjährigen Prozess erarbeitet. Dabei war die Pflege durch zwei, die Ärzteschaft durch sieben Expertinnen bzw. Experten unterschiedlicher Fachdisziplinen vertreten, daneben die Bereiche Recht, Psychologie, Seelsorge und Ethik durch jeweils eine Fachperson. Die Subkommission traf sich insgesamt zu 12 Sitzungen (darunter eine zweitägige Klausursitzung), konnte im Verlauf dieses Prozesses Experten einbeziehen und eine schriftliche Vorvernehmlassung durchführen, bei welchem Vertreterinnen und Vertreter des SBK, der FMH, Spezialisten der Palliativmedizin, des Rechts und der Allgemeinmedizin Stellung nehmen konnten. Schliesslich wurden die Richtlinien in der Zentralen Ethikkommission, dem Senat und dem Vorstand der SAMW intensiv diskutiert und überarbeitet.
Das hauptsächliche Anliegen der Richtlinien besteht darin, Aufgaben, Möglichkeiten und Grenzen der Betreuung von Patienten am Lebensende aufzuzeigen. Dabei besteht im Sinne der palliativen Betreuung das Hauptziel aller Massnahmen in der Linderung von Leiden und der Erhaltung der bestmöglichen Lebensqualität der Patienten sowie der Unterstützung der Angehörigen.

Bei aller Klarheit hinsichtlich des Hauptziels werden durch die Richtlinien eine Reihe schwieriger und teilweise auch umstrittener Themenbereiche geregelt. Hervorzuheben sind insbesondere:

- Die Bestimmung des Geltungsbereichs: Wie ist die Sterbephase klar genug von Situationen schwerkranker Menschen abzugrenzen, bei deren Betreuung unter Umständen andere Prioritäten zu setzen sind?

- Die Entscheidungsfindung bei nicht urteilsfähigen Patienten: Wie können Entscheidungen getroffen werden, wenn der mutmassliche Wille des Sterbenden nur ansatzweise oder überhaupt nicht bekannt ist?

- Die Verwirklichung der palliativen Betreuung: Was ist darunter genauer zu verstehen und wie lässt sich diese verwirklichen?

- Die Einschätzung der indirekten Sterbehilfe: Wie sieht ein angemessener Umgang mit schmerzlindernden Behandlungen bis hin zur Möglichkeit der palliativen Sedierung aus?

- Die Regelung der ärztlichen Beihilfe zum Suizid: Wie weit kann ein Arzt im Einzelfall gehen, wo sind die Grenzen zu setzen?

- Die Bereitstellung der nötigen Ressourcen: Werden de facto genügend Mittel zur Verfügung gestellt, um auch gemäss diesen Richtlinien handeln zu können?

Wichtige Akzente in der Beantwortung dieser komplexen Fragen wurden mit der klaren $A b$ grenzung des Geltungsbereichs gesetzt, welche Situationen schwerleidender Menschen ausserhalb der Sterbephase nicht mit einbeziehen, darüber hinaus mit der Betonung der Selbstbestimmung des Patienten und der starken Gewichtung einer rechtzeitig einsetzenden und umfassenden palliativen Betreuung. Die Berücksichtigung der 
Patientenautonomie hat nicht zuletzt auch zu einer gewissen Öffnung in bezug auf die Beurteilung der ärztlichen Beihilfe zum Suizid beigetragen: Auf der einen Seite ist die Beihilfe zum Suizid nicht Teil der ärztlichen Tätigkeit, denn der Arzt ist verpflichtet, seine ärztlichen Kompetenzen zur Heilung, Linderung und dem Beistehen einzusetzen. Auf der anderen Seite hat er den Willen des Patienten zu achten. Das kann auch bedeuten, dass eine persönliche Gewissensentscheidung des Arztes, im Einzelfall Beihilfe zum Suizid zu leisten, zu respektieren ist. Der einzelne Arzt trägt dann die Verantwortung für die Prüfung der nachfolgenden Mindestanforderungen, die in der heutigen Praxis offenkundig nicht immer eingehalten werden, namentlich das nahe bevorstehende Lebensende, die Frage, ob alternative Behandlungsmöglichkeiten erörtert und, soweit gewünscht, auch ausgeschöpft wur- den und ob der Wunsch aufgrund eines freien Entscheids und nicht durch Druck von aussen zustande kam.

Die Arbeit in der Subkommission war von dem gegenseitigen Willen geprägt, die zum Teil sehr unterschiedlichen Perspektiven zu verstehen und Richtlinien zur Vernehmlassung vorzulegen, die trotz unterschiedlicher Werthaltungen klare Orientierung $\mathrm{zu}$ geben und Leitplanken zu setzen imstande sind. Dass die Kommission sich diese Arbeit nicht leicht gemacht und um so manche Aussage ausführlich debattiert und gerungen hat, dürfte an einigen Formulierungen deutlich werden. Die Ärzteschaft ist eingeladen, im Rahmen der Vernehmlassung zu diesen wichtigen Richtlinien Rückmeldungen einzubringen (SAMW, Petersplatz 13, 4051 Basel, oder E-Mail: mail@samw.ch). 